\title{
Compromise of a-Defensin Function in Liver Cirrhosis Facilitates the Toxic Relationship Between Gut Permeability and Endotoxemia
}

\author{
Kanakaraju Kaliannan ${ }^{1}$
}

Published online: 14 July 2018

○) Springer Science+Business Media, LLC, part of Springer Nature 2018
Innate and adaptive immune dysfunction, also referred to as cirrhosis-associated immune dysfunction syndrome (CAIDS), is a major component of liver cirrhosis (LC), substantially contributing to the pathogenesis of the acute and chronic deterioration of liver function [1]. In advanced LC with CAID, small intestinal bacterial overgrowth (SIBO) and increased intestinal permeability (IP) are frequent findings linked to the translocation of bacteria and bacterial components such as endotoxin (lipopolysaccharide; LPS) from gut lumen to the systemic circulation, termed bacterial translocation (BT) [2]. BT is presumed to be the major mechanism leading to the development of spontaneous infections in liver cirrhosis, occurring in up to $30 \%$ of patients with decompensated LC, associated with a high mortality [3]. Despite the significant clinical burden, the precise underlying cellular and molecular pathways implicated in the phenomenon of BT in LC have not yet been studied.

Components of the hepatic immunological defense system include the innate immune system, exemplified by antimicrobial peptides (AMPs), considered the first line of defense against invading bacteria or their associated products [4]. LC also affects innate immunity by impairing the synthesis and function of pattern recognition receptors (PRRs) such as the toll-like receptors and related proteins that recognize LPS and other bacterial-derived molecules, inducing inflammatory cascades [4]. Gut-associated lymphoid tissue (GALT) is a component of mucosa-associated lymphoid tissue (MALT) that is the part of the immune system that defends against bacterial invasion of the intestine [4]. The adaptive immune system (AIS) is composed of highly specialized circulating lymphocytes (e.g., B cells and T cells)

Kanakaraju Kaliannan

kkaliannan@mgh.harvard.edu

1 Laboratory for Lipid Medicine and Technology, Department of Medicine, Massachusetts General Hospital and Harvard Medical School, 149 13th Street, Charlestown, MA 02129, USA that process and eliminate pathogens or prevent their multiplication. Nouri-Aria et al. [5] demonstrated a broad defect of T cells and hyperactivity of B cells in patients with LC.

AMPs and GALT are two major barriers that prevent BT [4]. Salzman et al. [6] have demonstrated that Paneth cell defensins can inhibit BT in a transgenic mouse model. Teltschik et al. suggested that intestinal mucosal barrier antimicrobial defenses are compromised in LC, increasing BT in experimental models of cirrhosis, although direct evidence supporting AMPs and GALT function in bacterial defense is lacking in humans, particularly when studied in individual intestinal segments. Likewise, the contribution of intestinal $\mathrm{T}$ cells is poorly defined in LC, although deserving of more attention since T cells impair BT translocation. Similarly, the relationship between intestinal AMPs and gut-derived endotoxemia in LC is not completely understood.

AMPs include defensins, cathelicidins, resistin-like molecules, bactericidal-permeability-inducing proteins, and lectins [4]. Among defensins, only $\alpha$ - and $\beta$-defensins have been identified in the alimentary tract. All mature defensins have broad-range antimicrobial activity achieved by disrupting the structure and function of microbial membranes. Neutrophils and Paneth cells strategically located at the base of the intestinal crypt predominantly express $\alpha$-defensins such as the human defensin (HD)5 and HD6 genes in humans [4]. Intestinal HD5 provides broad-spectrum microbicidal activity, whereas HD6 impedes the attachment of potentially pathogenic luminal organisms to the intestinal epithelial cells in order to successfully prevent enteric infection, thus contributing to the intestinal innate mucosal immunity. Moreover, the deficit in $\alpha$-defensins was accompanied by diminished in vitro antibacterial activity against several species of LPS-containing Enterobacteriaceae [7].

In this issue of Digestive Disease Science, Thomopoulos's group from University Hospital of Patras [8] demonstrates an association between compromised intestinal $\alpha$-defensins, GALT, and endotoxemia (a marker of intestinal barrier dysfunction and BT) [4]. The Paneth cells from patients with 


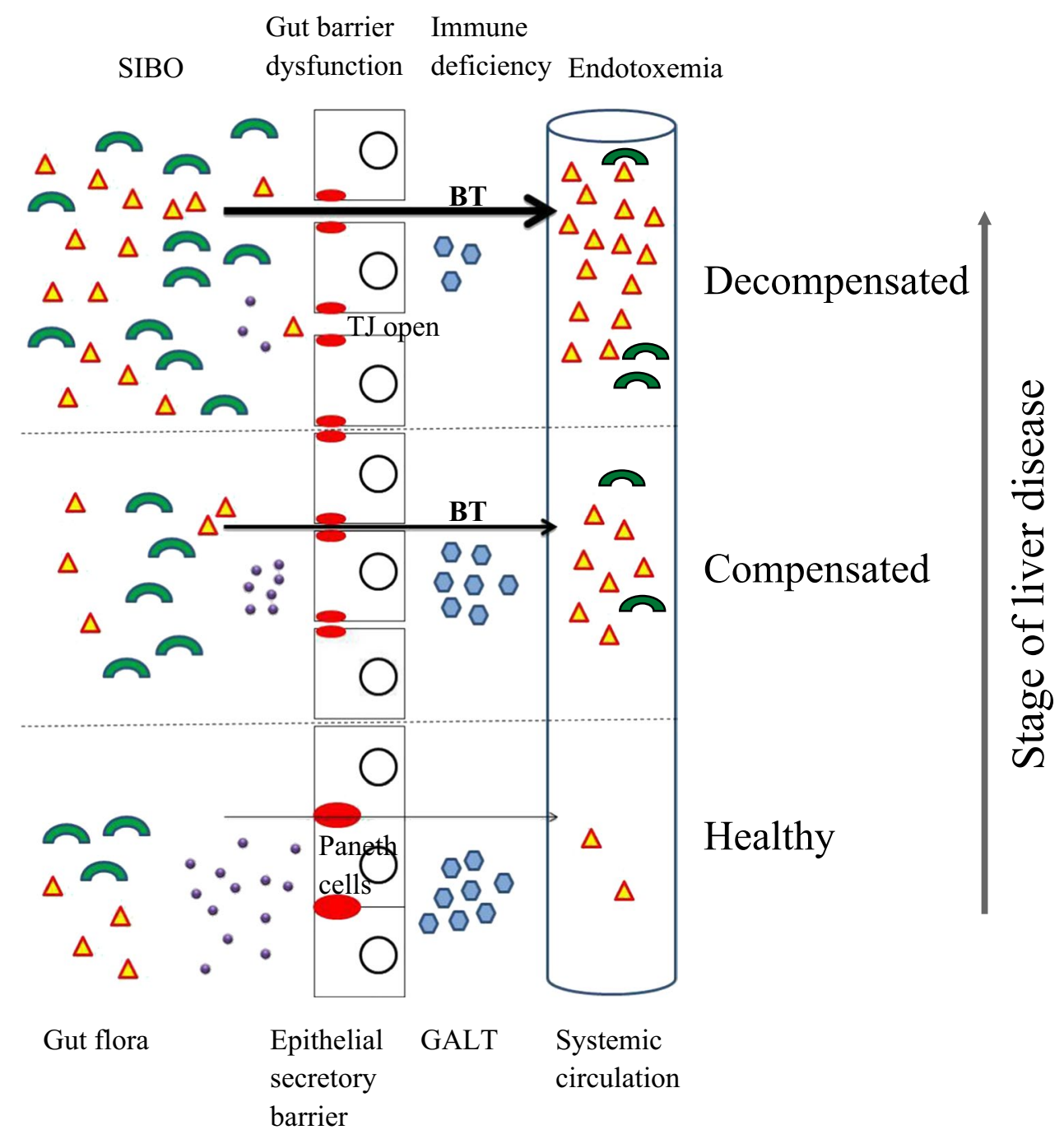

- Antimicrobial peptides (HD5/HD6)

$\triangle$ Vital bacteria
$\triangle$ Microbial products (e.g. endotoxins)
$\square$ T-lymphocytes
Tight junction (TJ)

Fig. 1 Stages of liver disease and a schematic depiction of a hypothesis addressing the evolution of pathologic bacterial translocation and endotoxemia in liver cirrhosis

LC appear to be depleted of granules as compared to normal controls. They discovered reduced HD5 and HD6 expression in duodenal Paneth cells and higher serum endotoxin levels in patients with LC compared to healthy controls, findings that were heightened in subjects with advanced stages of liver disease. Most importantly, HD5 and HD6 expression was inversely correlated with circulating endotoxin levels [8]. Moreover, they found that among patients with decompensated liver disease, endotoxin concentration was increased further in patients with higher Model of End-stage Liver Disease-Sodium (MELD-Na) score and higher ascites grade [8]. Therefore, a deficiency in $\alpha$-defensins likely decreases mucosal killing activity with a consequent shift of the luminal bacterial composition associated with SIBO 
and increased BT in LC. Meanwhile, the novel discovery of Tsiaoussis et al. [8] that depleted intraepithelial CD3+ T-lymphocytes (located within the epithelium, above the basal lamina, and between epithelial cells of duodenum) in advanced LC represents a defective ability of T-lymphocytes to migrate to the frontiers of the gut mucosal barrier and combat the pathogenic invaders. This is an important discovery since spontaneous BT of commensals occurs in the absence of $\mathrm{T}$ cells. Moreover, $\mathrm{T}$ cell depletion is associated with accumulation of bacteria in mesentric lymph nodes and the spreading of bacteria to extraintestinal sites in healthy rats [9].

All of these findings from this study inform a hypothesis that the development and progression of BT parallels the severity of liver disease (schematized in Fig. 1). In early stages, mild but constant occurrence of BT triggers an augmented pro-inflammatory cytokine response within the GALT that enhances bacterial defense mechanisms that further enhances intestinal barrier dysfunction, perpetuating and also adapting the immune system to increased BT. Ultimately, in decompensated cirrhosis, secretion of $\alpha$-defensins decreases, SIBO occurs, the function of T-lymphocytes within the GALT declines, and the amount of BT further increases. Subsequently, immune cell deficiency within the GALT with increased BT could then perpetuate the vicious cycle of inflammation accompanied by increased gut permeability and BT.

This would have been a more comprehensive study if the authors had provided data supporting the presence of SIBO, the status of other AMPs such as $\beta$-defensins, the expression of PRRs such as TLR-4, the measurement of intestinal inflammatory markers, had included more direct markers of BT such as serum bacterial genomic DNA, and had measured tight junctional protein expression and transepithelial permeability [10] in the duodenal biopsies.

Considering the close associations between intestinal barrier dysfunction, BT, and endotoxemia in $\mathrm{LC}$, the investigation of how host defense mechanisms are disrupted is of distinct clinical relevance. The novel discovery of Tsiaoussis et al. [8] opens new perspectives in microbiology, gut physiology and immunology. First, the mechanisms responsible for the observed compromised $\alpha$-defensin expression in different stages of LC are poorly understood. Second, more is to be discovered regarding the effect of $\alpha$-defensins on the expression of intestinal tight junction proteins and luminal microbial composition. Third, early assessment of immune dysfunction using routinely available methods can assist physicians involved in everyday clinical decision-making help design treatment strategies for patients with advanced liver disease. Fourth, dietary modulation of gut permeability, $\alpha$-defensin activity, and GALT functions [11] may form the basis for a promising approach for minimizing $\mathrm{BT}$ in $\mathrm{LC}$ patients.

\section{References}

1. Noor MT, Manoria P. Immune dysfunction in cirrhosis. J Clin Transl Hepatol. 2017;5:50-58.

2. Ponziani FR, Zocco MA, Cerrito L, et al. Bacterial translocation in patients with liver cirrhosis: physiology, clinical consequences, and practical implications. Expert Rev Gastroenterol Hepatol. 2018;12:641-656.

3. Cirera I, Bauer TM, Navasa M, et al. Bacterial translocation of enteric organisms in patients with cirrhosis. J Hepatol. 2001;34:32-37.

4. Wiest R, Lawson M, Geuking M. Pathological bacterial translocation in liver cirrhosis. J Hepatol. 2014;60:197-209.

5. Nouri-Aria KT, Alexander GJ, Portmann BC, et al. T and B cell function in alcoholic liver disease. J Hepatol. 1986;2:195-207.

6. Salzman NH, Ghosh D, Huttner KM, et al. Protection against enteric salmonellosis in transgenic mice expressing a human intestinal defensin. Nature. 2003;422:522-526.

7. Teltschik Z, Wiest R, Beisner J, et al. Intestinal bacterial translocation in rats with cirrhosis is related to compromised Paneth cell antimicrobial host defense. Hepatology. 2012;55:1154-1163.

8. Tsiaoussis GI, Papaioannou EC, Kourea EP, et al. Expression of alpha-Defensins, CD20+ B-lymphocytes, and Intraepithelial CD3+ T-lymphocytes in the Intestinal Mucosa of Patients with Liver Cirrhosis: Emerging Mediators of Intestinal Barrier Function. Dig Dis Sci. (Epub ahead of print). https://doi.org/10.1007/ s10620-018-5146-9.

9. Choudhry MA, Fazal N, Goto M, et al. Gut-associated lymphoid $\mathrm{T}$ cell suppression enhances bacterial translocation in alcohol and burn injury. Am J Physiol Gastrointest Liver Physiol. 2002;282:G937-G947.

10. Larsen R, Mertz-Nielsen A, Hansen MB, et al. Novel modified Ussing chamber for the study of absorption and secretion in human endoscopic biopsies. Acta Physiol Scand. 2001;173:213-222.

11. Kaliannan K, Wang B, Li XY, et al. A host-microbiome interaction mediates the opposing effects of omega- 6 and omega- 3 fatty acids on metabolic endotoxemia. Sci Rep. 2015;5:11276. 